MATEC Web of Conferences 44, 02051 (2016)

DOI: $10.1051 /$ matecconf/20164402051

(C) Owned by the authors, published by EDP Sciences, 2016

\title{
New method for solving the bending problem of rectangular plates with mixed boundary conditions
}

\author{
Xin Min Liu, Zhou Lei and Jing Yao Wu \\ College of Civil Engineering and Mechanics, Yanshan University, Qinhuangdao, China
}

\begin{abstract}
A new method is used to solve the rectangular plate bending problem with mixed boundary conditions. The method overcomes the complicated derivation of the classical solution by Fourth-order differential problem into integrating question. Under uniform loading rectangular plate bending problem with one side fixed the opposite side half simply supported half fixed the other two sides free rectangular plate, one side simply supported the opposite side half simply supported half fixed the other two sides free rectangular plate is systematically solved. According to the actual boundary conditions of the rectangular plate, the corresponding characteristic equation can easily be set up. It is presented deflection curve equation and the numerical calculation. By compared the results of the equation to the finite element program, we are able to demonstrate the correctness of the method. So the method not only has certain theoretical value, but also can be directly applied to engineering practice.
\end{abstract}

\section{Introduction}

With the development of the construction industry, the diversity of building is in high demand. It is more and more important to calculate plates under various constraint conditions. So in this paper, applying a new method to solve the bending problem of rectangular plates with mixed boundary conditions .The method provides a simple and practical calculation method for engineering calculation of rectangular plates with mixed boundary conditions, and it has certain theoretical and engineering significance. The method is no need to consider the hypothesis of complex displacement function, which can be very easy to write the total potential energy of rectangular plate on mixed boundary conditions. According to the actual boundary conditions of rectangular plate, corresponding characteristic equation can be easily established, thereby to overcome the cumbersome derivation process in classic solution. Because this method is simple and program characteristics, especially which is more efficient to solve the other methods are not easy to solve problems.

\section{Theoretical basis for the new method}

Static equilibrium equation of bending plates [1-2]

$$
\frac{\partial^{4} w}{\partial x^{4}}+2 \frac{\partial^{4} w}{\partial x^{2} \partial y^{2}}+\frac{\partial^{4} w}{\partial y^{4}}=\frac{q}{D}
$$

Fourth-order differential problem is converted to integrating question due to the complicated derivation of the classical solution.
According to the generalized virtual work principle [3], there is

$$
\begin{aligned}
\iiint_{V} \sigma_{i j} \delta e_{i j} d V= & \iiint_{V} F_{i} \delta u_{i} d V+\iint_{S_{p}} \overline{p_{i}} \delta u_{i} d S \\
& +\iint_{S_{u}} p_{i} \delta \bar{u}_{i} d S
\end{aligned}
$$

Or according to the known boundary displacement variation potential energy principle [4]

$$
\begin{aligned}
\iiint_{V} \delta A(e) d V= & \iiint_{V} F_{i} \delta u_{i} d V+\iint_{S_{p}} \overline{p_{i}} \delta u_{i} d S \\
& +\iint_{S_{u}} p_{i} \delta \bar{u}_{i} d S
\end{aligned}
$$

Outside surface Yu Gong zero variation principle [5-7]

$$
\iint_{s_{u}} p_{i} \delta \bar{u}_{i} \mathrm{~d} S=-\iint_{s_{u}} \bar{u}_{i} \delta p_{i} \mathrm{~d} S
$$

Substituting (3), then

$$
\begin{aligned}
\iiint_{V} \delta A(e) d V= & \iiint_{V} F_{i} \delta u_{i} d V+\iint_{S_{p}} \overline{p_{i}} \delta u_{i} d S \\
& -\iint_{S_{u}} \bar{u}_{i} \delta p_{i} d S
\end{aligned}
$$

According to formula (5) can be obtained

$$
\begin{gathered}
\Pi_{m p}=\iiint_{{ }_{V}} \delta A(e) \mathrm{d} V-\iiint_{V} F_{i} \delta u_{i} \mathrm{~d} V- \\
\iint_{S_{p}} \overline{p_{i}} \delta u_{i} \mathrm{~d} S+\iint_{S_{u}} \bar{u}_{i} \delta p_{i} \mathrm{~d} S
\end{gathered}
$$

Zhou Lei:604167145@qq.com 
Type (6) is called total potential energy of mixed variable potential energy principle [8], or simply mixed total potential energy.

Take extreme variation for $u_{i}$ and $p_{i}$ in the formula (6), then we have

$$
\begin{array}{r}
\delta \prod_{m p}=\iiint_{{ }_{V}} \delta A(e) \mathrm{d} V-\iiint_{{ }_{V}} F_{i} \delta u_{i} \mathrm{~d} V- \\
\\
\qquad \int_{S_{p}} \overline{p_{i}} \delta u_{i} \mathrm{~d} S+\iint_{S_{u}} \bar{u}_{i} \delta p_{i} \mathrm{~d} S=0
\end{array}
$$

Pay attention to

$$
\begin{gathered}
\iiint_{V} \delta A(e) d V=\iiint_{V} \frac{\delta A(e)}{\partial e_{i j}} \delta e_{i j} d V=\iiint_{V} \sigma_{i j} \delta e_{i j} d V \\
=\iiint_{V} \sigma_{i j} \delta u_{i, j} d V=\iiint_{V}\left[\left(\sigma_{i j} \delta u_{i}\right)_{, j}-\sigma_{i j, j} \delta u_{i}\right] d V \\
=\iint_{S} \sigma_{i j} n_{j} \delta u_{i} d S-\iiint_{V} \sigma_{i j, j} \delta u_{i} d V \\
=\iint_{S_{p}} \sigma_{i j} n_{j} \delta u_{i} d S+\iint_{S_{U}} \sigma_{i j} n_{j} \delta u_{i} d S \\
-\iint_{V} \sigma_{i j, j} n_{j} \delta u_{i} d S
\end{gathered}
$$

Mixed total potential energy is required that the displacement is weakly allowed and the boundary force is coordination allowed [9]. According to this definition, the $\delta u_{i}$ is not zero on $S_{u}$. Type formula (8) into (7), then

$$
\begin{aligned}
\delta \prod_{m p}= & -\iiint_{V}\left(\sigma_{i j, j}+F_{i}\right) \delta u_{i} \mathrm{~d} V+\iint_{s_{P}}\left(\sigma_{i j} n_{j}-\bar{p}_{i}\right)_{i} \delta u_{i} \mathrm{dS} \\
& +\iint_{s_{u}} \sigma_{i j} n_{j} \delta u_{i} \mathrm{~d} S+\iint_{s_{u}} \bar{u}_{i} \delta p_{i} \mathrm{~d} S=0
\end{aligned}
$$

According to the inner surface $\mathrm{Yu}$ Gong zero variation principle

$$
\iint_{S_{u}} \sigma_{i j} n_{j} \delta u_{i} \mathrm{~d} S=-\iint_{S_{u}} u_{i} \delta\left(\sigma_{i j} n_{j}\right) \mathrm{d} S
$$

Formula (9) becomes

$$
\begin{aligned}
\delta \prod_{m p}= & -\iiint_{V}\left(\sigma_{i j, j}+F_{i}\right) \delta u_{i} \mathrm{~d} V+\iint_{S_{P}}\left(\sigma_{i j} n_{j}-\bar{p}_{i}\right)_{i} \delta u_{i} \mathrm{dS} \\
& -\iint_{S_{u}}\left(u_{i}-\bar{u}_{i}\right) \delta p_{i} \mathrm{~d} S=0
\end{aligned}
$$

Taking extreme variation on the displacement and force can obtain the corresponding deflection curve equation.

\section{One side fixed the opposite side half simply supported half fixed the other two sides free rectangular plate}

Take only by the unit concentrated force simply supported rectangular plate as a basic system, as shown in Fig.1. To calculate the curved rectangular plate, taking one side fixed the opposite side half simply supported half fixed the other two sides free under uniform loads rectangular plate as the actual system, as shown in Fig.2.

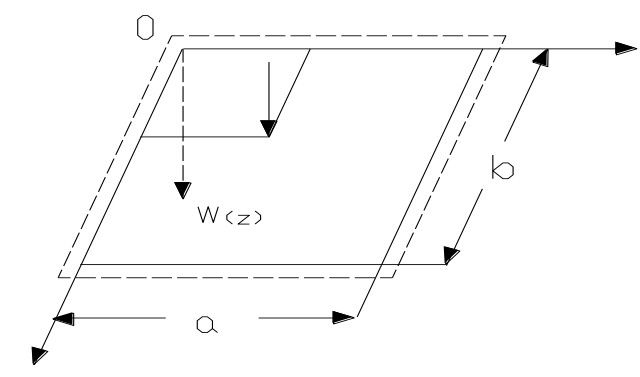

Figure 1. Virtual basic system for thin plate

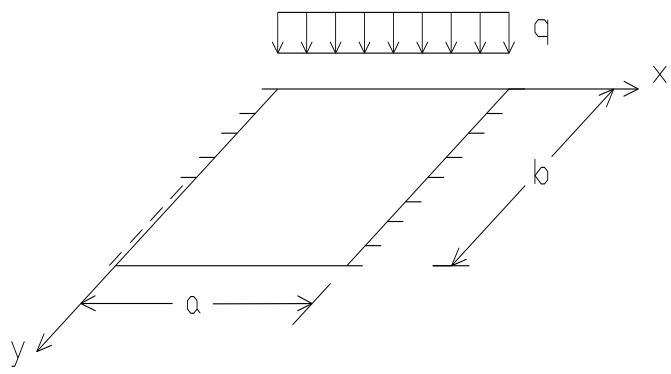

Figure 2. Actual system

\subsection{Deflection surface equation}

For the actual system Fig 2, fixed side bending constraint is released and this constraint is replaced by distributed moments, as shown in Fig 3.
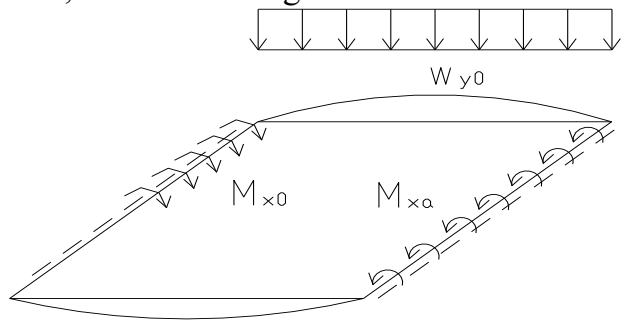

Figure 3.Actual system of fixed side bending constraints relesed

$$
\begin{array}{ll}
M_{x 0}=\sum_{n=1,2}^{\infty} A_{n} \sin \beta_{n} y & 0<y<\mathrm{b} \\
M_{x a}=\sum_{n=1,2}^{\infty} B_{n} \sin \beta_{n} y & 0<y<\mathrm{b} / 2
\end{array}
$$

$\mathrm{y}=0, \mathrm{y}=\mathrm{b}$ side deflection is:

$$
\begin{aligned}
& W_{y 0}=\sum_{n=1,2}^{\infty} C_{m} \sin \alpha_{m} x \\
& W_{y b}=\sum_{m=1,2}^{\infty} d_{m} \sin \alpha_{m} x
\end{aligned}
$$

The new method can get one side fixed the opposite side half simply supported half fixed the other two sides free rectangular plate total potential energy of minimum potential energy principle, such as type (16) 


$$
\begin{aligned}
& \prod_{m p}= \int_{0}^{a} \int_{0}^{b} \frac{1}{2} D\left(\frac{\partial^{2} w}{\partial x^{2}}+\frac{\partial^{2} w}{\partial y^{2}}\right)^{2} d x d y- \\
& \int_{0}^{a} \int_{0}^{b} \frac{2 D(1-v)}{2}\left[\frac{\partial^{2} w}{\partial x^{2}} \frac{\partial^{2} w}{\partial y^{2}}-\left(\frac{\partial^{2} w}{\partial x \partial y}\right)^{2}\right] d x d y \\
&-\int_{0}^{a} \int_{0}^{b} q w d x d y-\int_{0}^{\frac{b}{2}} \bar{M}_{x 0}\left(\frac{\partial w}{\partial x}\right)_{x 0} d y+ \\
& \int_{0}^{b} \bar{M}_{x a}\left(\frac{\partial w}{\partial y}\right)_{x a} d y+\int_{0}^{a} w_{y b} V_{y b} d x-\int_{0}^{a} w_{y 0} V_{y 0} d x
\end{aligned}
$$

The formula (12) - (15) into equation (16), where taking $\prod_{m p}$ extreme variation to get deflection surface equation:

$$
\begin{gathered}
W(\xi, \eta)=\int_{0}^{a} \int_{0}^{b} q w_{1} d x d y+\int_{0}^{\frac{b}{2}} M_{x 0} w_{1 x 0} d y- \\
\int_{0}^{b} M_{x a} w_{1 x a} d y+\int_{0}^{a} V_{1 y 0} w_{y 0} d y-\int_{0}^{a} V_{1 y b} w_{y b} d x+R_{1 a b} k_{3} \\
=w_{1}+w_{2}+w_{3}+w_{4}+w_{5}
\end{gathered}
$$

Among them:

$$
\begin{aligned}
& w_{1}=\frac{4 q}{D a} \sum_{m=1,3}^{\infty}\left\{1+\frac{1}{2 c h \frac{1}{2} \alpha_{m} b}\left[\alpha_{m}\left(\eta-\frac{b}{2}\right) \operatorname{sh} \alpha_{m}\left(\eta-\frac{b}{2}\right)\right.\right. \\
& \left.\left.-\left(2+\frac{1}{2} \alpha_{m} b t h \frac{1}{2} \alpha_{m} b\right) \operatorname{ch} \alpha_{m}\left(\eta-\frac{b}{2}\right)\right]\right\} \cdot \frac{1}{\alpha_{m}^{5}} \sin \alpha_{m} \xi \\
& =\frac{4 q}{D a} \sum_{n=1,3}^{\infty}\left\{1+\frac{1}{2 \operatorname{ch} \frac{1}{2} \beta_{n} a}\left[\beta_{n}\left(\zeta-\frac{a}{2}\right) \operatorname{sh} \beta_{n}\left(\zeta-\frac{a}{2}\right)-\right.\right. \\
& \left.\left.\left(2+\frac{1}{2} \beta_{n} a t h \frac{1}{2} \beta_{n} a\right) \operatorname{ch} \beta_{n}\left(\zeta-\frac{a}{2}\right)\right]\right\} \cdot \frac{1}{\beta_{n}^{5}} \sin \beta_{n} \eta(18) \\
& w_{2}=\frac{1}{4 D} \sum_{n=1,2}^{\infty}\left(-\frac{\beta_{n} a}{s h^{2} \beta_{n} a} \operatorname{sh} \beta_{n} \xi+c t h \beta_{n} a \beta_{n} \xi \operatorname{ch} \beta_{n} \xi\right) \\
& \frac{A_{n}}{\beta_{n}^{2}} \sin \beta_{n} \eta-\frac{1}{4 D} \sum_{n=1,2}^{\infty} \beta_{n} \xi \operatorname{sh} \beta_{n} \xi \frac{A_{n}}{\beta_{n}^{2}} \sin \beta_{n} \eta \\
& w_{4}=\frac{1}{2} \sum_{n=1,2}^{\infty} 2 \frac{C_{m}}{\beta_{n}^{2} s h \alpha_{m} b} \operatorname{sh} \alpha_{m}(b-\eta) \sin \alpha_{m} \xi+ \\
& w_{0}^{b} M_{x a} w_{1 x a} d y \frac{1}{2} \sum_{n=1,2}^{\infty}\left(\beta_{n} a c t h \beta_{n} a-\beta_{n} \xi \operatorname{cth} \beta_{n} \xi\right)
\end{aligned}
$$

$$
\begin{gathered}
-D\left[\frac{\partial^{3} w}{\partial \eta^{3}}+(2-v) \frac{\partial^{3} w}{\partial^{2} \xi \partial \eta}\right]_{\eta=b}=0 \\
-D\left[\omega_{1 \eta \eta \eta}^{\prime \prime \prime}+\omega_{2 \eta \eta \eta}^{\prime \prime \prime}+\omega_{3 \eta \eta \eta}^{\prime \prime \prime}+\omega_{4 \eta \eta \eta}^{\prime \prime \prime}+\omega_{5 \eta \eta \eta}^{\prime \prime \prime}+(2-v)\right. \\
\left..\left(\omega_{1 \xi \xi \eta}^{\prime \prime \prime}+\omega_{2 \xi \xi \eta}^{\prime \prime \prime}+\omega_{3 \xi \xi \eta}^{\prime \prime \prime}+\omega_{4 \xi \xi \eta}^{\prime \prime \prime}+\omega_{5 \xi \xi \eta}^{\prime \prime \prime}\right)\right]_{\eta=b}=0
\end{gathered}
$$

$$
\begin{aligned}
& -D\left[\omega_{1 \eta \eta \eta}^{\prime \prime \prime}+\omega_{2 \eta \eta \eta}^{\prime \prime \prime}+\omega_{3 \eta \eta \eta}^{\prime \prime \prime}+\omega_{4 \eta \eta \eta}^{\prime \prime \prime}+\omega_{5 \eta \eta \eta}^{\prime \prime \prime}+(2-v)\right. \\
& \left..\left(\omega_{1 \xi \xi \eta}^{\prime \prime \prime}+\omega_{2 \xi \xi \eta}^{\prime \prime \prime}+\omega_{3 \xi \xi \eta}^{\prime \prime \prime}+\omega_{4 \xi \xi \eta}^{\prime \prime \prime}+\omega_{5 \xi \xi \eta}^{\prime \prime \prime}\right)\right]_{\eta=0}=0
\end{aligned}
$$

Infinite equations are derived through taking the deflection surface equation into the corresponding boundary conditions of the formula (23) to formula (30) .It is obtained unknown number through assigning for equations.

\subsection{Numerical calculation}

Calculation parameters were taken as $\mathrm{a}=\mathrm{b}=1 \mathrm{~m}$, $\mathrm{q}=100 \mathrm{~N} / \mathrm{m}^{2}, \mathrm{E}=200 \mathrm{GPa}, \mathrm{h} / \mathrm{a}=0.005 、 0.1 、 0.2 、 0.3$, $\mathrm{v}=0.3$. Since the trigonometric convert to hyperbolic 
functions makes infinite series converges issue is resolved so $\mathrm{m}=\mathrm{n}=1,2,3 \ldots 50$. By using MATLAB software programming for the $A_{n}, C_{m}, B_{n}, d_{m}$ and deflection expression. For example, $\mathrm{h} / \mathrm{a}=0.3$ compared with the calculation results of ANSYS, both tend to be consistent, the error is less than $5 \%$.

Table 1. Under uniform loading for one side fixed the opposite side half simply supported half fixed the other two sides free rectangular plate deflection value

\begin{tabular}{ccccccccccccc}
\hline $\mathrm{X}$ & $\mathrm{y}=0.00$ & $\mathrm{y}=0.00$ & $\mathrm{y}=0.25$ & $\mathrm{y}=0.25$ & $\mathrm{y}=0.5$ & $\mathrm{y}=0.5$ & $\mathrm{y}=0.75$ & $\mathrm{y}=0.75$ & $\mathrm{y}=1$ & $\mathrm{y}=1$ \\
\cline { 2 - 6 } & Article & ANSYS & Article & ANSYS & Article & ANSYS & Article & ANSYS & Article & ANSYS \\
\hline 0.0 & 0.000000 & 0.000000 & 0.000000 & 0.000000 & 0.000000 & 0.000000 & 0.000000 & 0.000000 & 0.000000 & 0.000000 \\
\hline 0.1 & 0.000340 & 0.000331 & 0.000396 & 0.000376 & 0.000672 & 0.000666 & 0.001426 & 0.001390 & 0.001501 & 0.001591 \\
\hline 0.2 & 0.001115 & 0.001171 & 0.001234 & 0.001194 & 0.001563 & 0.001685 & 0.002538 & 0.002629 & 0.002796 & 0.002787 \\
\hline 0.3 & 0.001956 & 0.001985 & 0.002122 & 0.002047 & 0.002487 & 0.002598 & 0.003469 & 0.003559 & 0.003886 & 0.003879 \\
\hline 0.4 & 0.002582 & 0.002813 & 0.002773 & 0.002651 & 0.003079 & 0.003084 & 0.003978 & 0.004042 & 0.004197 & 0.004180 \\
\hline 0.5 & 0.002895 & 0.002934 & 0.003082 & 0.002986 & 0.003289 & 0.003269 & 0.004186 & 0.004150 & 0.004365 & 0.004299 \\
\hline 0.6 & 0.002581 & 0.002550 & 0.002699 & 0.002600 & 0.003036 & 0.002918 & 0.003896 & 0.003868 & 0.004162 & 0.004165 \\
\hline 0.7 & 0.001897 & 0.001916 & 0.002054 & 0.001972 & 0.002465 & 0.002373 & 0.003419 & 0.003537 & 0.003836 & 0.003848 \\
\hline 0.8 & 0.000997 & 0.001054 & 0.001196 & 0.001137 & 0.001585 & 0.001533 & 0.002491 & 0.002423 & 0.002787 & 0.002719 \\
\hline 0.9 & 0.000328 & 0.000324 & 0.000428 & 0.000456 & 0.000679 & 0.000682 & 0.000139 & 0.000143 & 0.001569 & 0.001501 \\
\hline 1.0 & 0.000000 & 0.000000 & 0.000000 & 0.000000 & 0.000000 & 0.000000 & 0.000000 & 0.000000 & 0.000000 & 0.000000 \\
\hline
\end{tabular}

\section{One side simply supported the opposite side half simply supported half fixed the other two sides free rectangular plate}

To calculate the curved rectangular plate, taking one side simply supported the opposite side half simply supported half fixed the other two sides free under uniform loads rectangular plate as the actual system, shown in Fig.4.

\subsection{Deflection surface equation}

For the actual system Fig 4, fixed side bending constraint is released and this constraint is replaced by distributed moment, as shown in Fig 5.

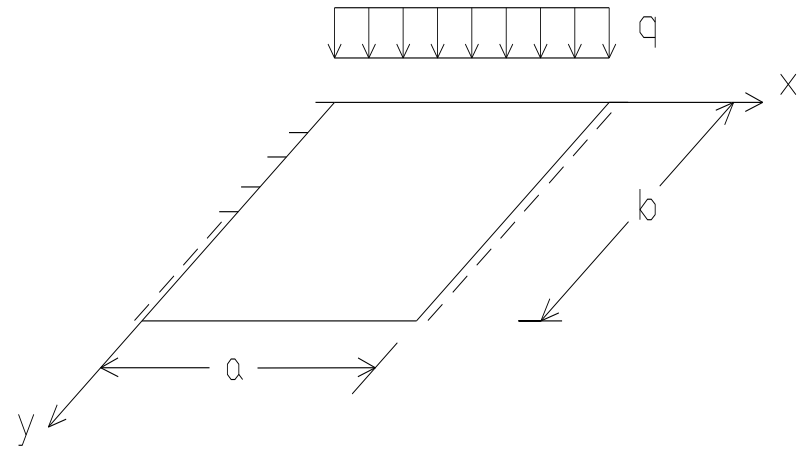

Figure 4. Actual system

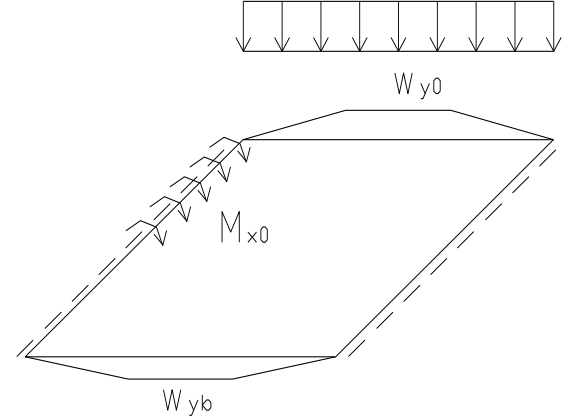

Figure 5. Actual system of fixed side bending constraints relesed

$$
M_{x 0}=\sum_{n=1,2}^{\infty} A_{n} \sin \beta_{n} y
$$

$\mathrm{y}=0, \mathrm{y}=\mathrm{b}$ side deflection is:

$$
\begin{aligned}
& w_{y 0}=\sum_{m=1,2}^{\infty} C_{m} \sin \alpha_{m} x \\
& w_{y b}=\sum_{m=1,2}^{\infty} d_{m} \sin \alpha_{m} x
\end{aligned}
$$

The new method can get one side simply supported the opposite side half simply supported half fixed the other two sides free rectangular plate total potential energy of minimum potential energy principle, such as type (34) 


$$
\begin{aligned}
\prod_{m p}= & \int_{0}^{a} \int_{0}^{b} \frac{1}{2} D\left(\frac{\partial^{2} w}{\partial x^{2}}+\frac{\partial^{2} w}{\partial y^{2}}\right)^{2} d x d y- \\
& \int_{0}^{a} \int_{0}^{b} \frac{2 D(1-v)}{2}\left[\frac{\partial^{2} w}{\partial x^{2}} \frac{\partial^{2} w}{\partial y^{2}}-\left(\frac{\partial^{2} w}{\partial x \partial y}\right)^{2}\right] d x d y \\
& -\int_{0}^{a} \int_{0}^{b} q w d x d y-\int_{0}^{\frac{b}{2}} \bar{M}_{x 0}\left(\frac{\partial w}{\partial x}\right)_{x 0} d y+ \\
& \int_{0}^{a} w_{y b} V_{y b} d x-\int_{0}^{a} w_{y 0} V_{y 0} d x
\end{aligned}
$$

The formula (31) - (33) into equation (34), where taking $\prod_{m p}$ extreme variation to get deflection surface equation:

$$
\begin{aligned}
W(\xi, \eta)= & \int_{0}^{a} \int_{0}^{b} q w_{1} d x d y+\int_{0}^{\frac{b}{2}} M_{x 0} w_{1 x 0} d y+ \\
& \int_{0}^{a} V_{1 y 0} \omega_{y o} d x-\int_{0}^{a} V_{1 y b} w_{y b} d x \\
= & w_{1}+w_{2}+w_{4}+w_{5}
\end{aligned}
$$

$w_{1}$ is the same as type (18); $w_{2}$ is the same as type (19); $w_{4}$ is the same as type (21); $w_{5}$ is the same as the type (22).

\subsection{Boundary conditions}

Deflection surface equation (35) must satisfy the following boundary conditions

$$
\begin{gathered}
\left(\frac{\partial w}{\partial \xi}\right)_{\xi=0}=0 \\
-D\left[\frac{\partial^{3} w}{\partial \eta^{3}}+(2-v) \frac{\partial^{3} w}{\partial \eta \partial \xi^{2}}\right]_{\eta=0}=0 \\
-D\left[\frac{\partial^{3} w}{\partial \eta^{3}}+(2-v) \frac{\partial^{3} w}{\partial \eta \partial \xi^{2}}\right]_{\eta=b}=0
\end{gathered}
$$

Infinite equations are derived through taking the deflection surface equation into the corresponding boundary conditions of the formula (36) to formula (38) .It is obtained unknown number through assigning for equations.

\subsection{Numerical calculation}

Calculation parameters were taken $\mathrm{a}=\mathrm{b}=1 \mathrm{~m}, \mathrm{q}=100 \mathrm{~N} / \mathrm{m} 2$, $\mathrm{E}=200 \mathrm{GPa}, \mathrm{h} / \mathrm{a}=0.005 、 0.1 、 0.2 、 0.3, \mathrm{v}=0.3$ 。 $\mathrm{m}=\mathrm{n}=1,2,3 \ldots 50$, by using MATLAB software programming for the $\mathrm{An}, \mathrm{Cm}, \mathrm{dm}$ and deflection expression. For example, $\mathrm{h} / \mathrm{a}=0.3$ compared with the calculation results of ANSYS, both tend to be consistent, the error is less than $5 \%$. The deflection curves of plate are showed in Fig.6.

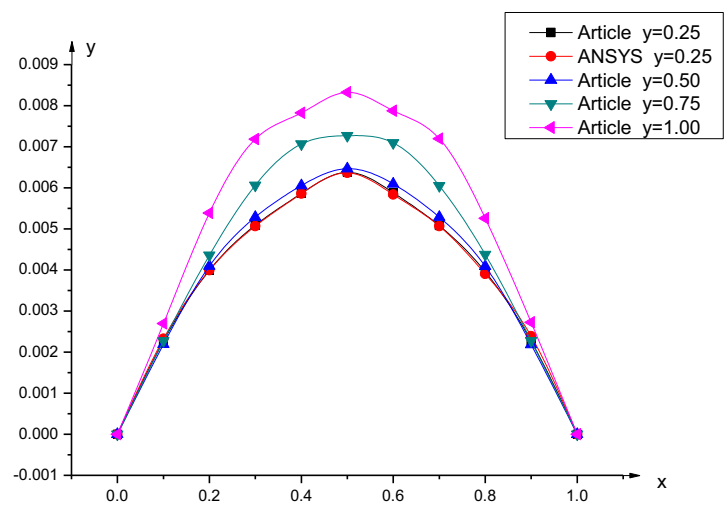

Figure 6. Virtual basic system for thin plate

\section{Conclusion}

In this paper, the new method is applied to solve the bending problem of rectangular plate with mixed boundary conditions under uniform loads. In the MATLAB platform, it is conducted numerical calculation for different issues. In addition, computational analysis of the rectangular plate is carried out using finite element software ANSYS. It is verified the accuracy of the numerical results by comparison, so that the new method is correct.

\section{References}

1. M. A. Leitao. A Meshless Method for Kirchhoff Plate Bending Problems. European Journal of Mechanics Solids, 2001, 52(10): 1107-1130.

2. Sewell, m.j. on Dual Approximation Principles and Optimization in Continuum Mechanics, phill. Trans. R. soc. London, 1969, (a-1162): 319-350.

3. FU BL, Energy principle and its application in elastic mechanics [M]. Beijing: Science Press, 2004:366401,498-582.

4. Luo JX, Li Q, Research on the relationship between the Ritz method and Galerkin method [J], Shanxi Architecture, 2007,33(10): 96-97.

5. Yu F, X. B. Liu. New Exact Solutions for Free Vibrations of Rectangular Thin Plates by Symplectic Dual Method. Acta Mechanica Sinica, 2009, (2): 265-270.

6. $\mathrm{Hu} \mathrm{XG}, \mathrm{Su} \mathrm{TJ}$. The minimum excess principle used in dynamic analysis of elastic circular plate. Jilin Normal University Newspaper (NATURAL SCIENCE EDITION), 2005, (2): 11-16.

7. Fu BL, Variational principle of extremum value with mixed variable in elasticity[J]. Journal of Xingtai Vocational and Technical College, 2003:1-9.

8. Fu BL, Principle of minimum potential energy with mixed variables and its application [J]. Journal of Yanshan University, 2011:1-8.

9. Zhang L, Bending beam alternative method for calculating internal forces [J], Journal of Jingdezhen College, 2008, 23(2): 6-7. 